phase where self-worth is often judged by how a person looks.

The aim of this study was to describe the spectrum of skin disease seen in school going adolescents in a developing country and evaluate the effect of these disorders on their quality of life using the Children's Dermatology Life Quality Index (CDLQI).

Methods Adolescents attending both private and public secondary schools in a developing country were recruited using a multi-staged stratified random sampling method. Socio-demographic details were recorded and they all underwent physical examination by paediatric dermatologists. All those with skin lesions were then administered the CDLQI and scores were recorded and analyzed as appropriate using SPSS version 20.

Results Out of the 1150 students recruited, 746 (64.9\%) had skin disorders. The prevalent skin disorders were acne vulgaris, pityriasis versicolor, tinea capitis, pityriasis capitis and traction alopecia which were responsible for $88.9 \%$ of skin disease diagnosed. Five hundred and forty-two $(72.6 \%)$ reported an impairment in their dermatology-specific Quality of Life and majority of this number had a mild effect on life quality. The domain of the CDLQI where students had the most impairment was the symptom/feeling domain, followed by impairment from treatment. Adolescents receiving private education were more likely to report impairment in their dermatology-specific Quality of life $(p<0.05)$.

Conclusion Skin disorder is common in Nigerian adolescents with a few handful accounting for the bulk of all dermatoses seen. It is mostly associated with an impaired dermatologyspecific quality of life, albeit a mild impairment. There's a need for more awareness and improved access to skin care for Nigerian adolescents.

\section{G615(P) BEWARE! THE EMERGING SUPERBUG}

MJ Lavery, K Macbeth, ML Walsh. Dermatology, St Helens and Knowsley Teaching Hospitals NHS Trust, St Helens, UK

\subsection{6/archdischild-2020-rcpch.529}

A fit and healthy 8-year old boy presented with a 10-day history of asymptomatic superficial ulcerations initially on the left great toe, and then the left elbow. He was systemically well. The general practitioner had prescribed oral flucloxacillin for infected bite marks but the patient took for 2-3 days as the parents felt some of the lesions were starting to resolve. However within days he developed new lesions on the left foot and toes and was admitted for intravenous antibiotics. On examination there was an exudative ulcerated papule on the dorsum of the left foot and left 3rd toe with surrounding erythema and oedema. Bacterial swab was positive for staphylococcus aureus (SA), and further analysis confirmed the clinical suspicion of panton-valentine-leukocidin SA (PVL-SA) positivity. The patient had a good clinical response to augmentin/clavulanic acid with subsequent decolonisation with chlorhexidine $4 \%$ body wash and nasal chlorhexidine with neomycin cream. Close contacts also received eradication therapy.

The prevalence of PVL-SA positive isolates from skin wounds is increasing. In the United Kingdom 20\% of SA isolates from skin and soft tissue were found to be PVL-SA positive (compared to $2 \%$ in 2005). Patients and/or close contacts present with either persistent purulent wounds resistant to usual treatments, or recurrent boils and abscesses. Risk factors include the 'five C's' - contaminated items, close contact, crowding, cleanliness and cuts or compromised skin integrity. The Health Protection Agency has produced guidance on the diagnosis and management of PLV-SA including eradication regimes, which our patient and close contacts received.

\section{G616(P) CUTANEOUS MANIFESTATIONS OF CHRONIC KIDNEY DISEASE IN PAEDIATRICS IN A TERTIARY CHILDREN'S HOSPITAL IN SUDAN}

${ }^{1} \mathrm{~L}$ Kheir Elseed, ${ }^{2} \mathrm{~A}$ Bakhet. ${ }^{1}$ Department of Dermatology and Venereology, Khartoum Dermatology and Cenereology Teaching Hospital, Khartoum, Sudan; ${ }^{2}$ Department of Dermatology and Venereology, Khartoum North Teaching Hospital, Khartoum, Sudan

\subsection{6/archdischild-2020-rcpch.530}

Background The skin is the largest immunological organ in the human body; it is the protective barrier of the body, so the skin changes can reflect the internal status of the body.

Many of the skin changes are known to occur in chronic kidney disease (CKD).

Several studies have evaluated cutaneous manifestations in adult patients with $\mathrm{CKD}$ on haemodialysis or conservative treatment worldwide, but few studies evaluated this in paediatric patients.

Objectives The aim of this study was to identify the prevalence and clinical pattern of skin manifestations among children with chronic kidney disease (CKD), and also to describe its relationship to haemodialysis and other variants: demographic, medical and laboratory values in children with CKD.

Methods The study included 50 patients, 30 were on haemodialysis and 20 patients on conservative treatment, in different stages of CKD. After history was taken and examinations was done, many parameters were evaluated in those patients including; age and gender, medications, duration of CKD and haemodialysis, causes and stages of CKD. Also, laboratory values included in the analysis were haemoglobin, calcium, urea and creatinine levels. Data was collected over a period of 6 months.

Results This study results showed skin changes were detected in 43 patients $(86 \%)$, and more than one change in the same patient was seen in 18 (36\%).

Skin changes noted were mainly: xerosis in 34 (68\%), Pruritus in 32 (64\%), hair loss in $15(30 \%)$ and hyperpigmentation in $14(28 \%)$ patients.

Other changes included eczema 1 (2\%), urticaria 1 (2\%), seborrheic dermatitis $1(2 \%)$ and lichen planus 1 (2\%).

The manifestations observed were not affected by age, gender nor by the precipitant cause of CKD.

There was a notable association between anaemia, uraemia and high serum creatinine and the presence of skin changes.

Conclusion

- Skin changes are common in children with CKD.

- These changes were mainly due to $\mathrm{CKD}$ rather than haemodialysis, and rarely due to drug treatment.

- Dermatological examination is important and useful and it can help point towards a diagnosis of CKD. 